\section{Breast cancer prophylaxis}

\section{By Michael J. Haas, Senior Writer}

Although the recent FDA approval of Dendreon Corp.'s Provenge therapeutic vaccine for prostate cancer finally has cemented immunotherapy's place in the cancer armamentarium, the development of prophylactic cancer vaccines has lagged far behind. One reason is that immunization with tumor antigens, which are derived from self-antigens, can lead to autoimmune responses that damage normal tissues-a risk that might be acceptable to cancer patients receiving a therapeutic vaccine but unacceptable to healthy individuals receiving a prophylactic one.

Researchers at the Cleveland Clinic and Cleveland State University think they have side-stepped this problem by showing that a vaccine based on $\alpha$-lactalbumin (LALBA) safely protected mice from breast cancer. ${ }^{1}$ Now comprehensive studies
“Most women over 40 don't plan to have more children and said the trade-off was not an issue at all."

-Vincent Tuohy, Cleveland Clinic
University and a staff member in the Department of Immunology at the clinic, led the researchers. The group published the results in Nature Medicine.

"The team has done great work here in preclinical models, and the vaccine appears interesting," said Brent Treiger, VP of clinical R\&D at Apthera Inc.

Apthera is developing NeuVax (E75), a vaccine consisting of an immunogenic peptide derived from HER2 (ERBB2; neu) and sargramostim. It is in Phase II/III testing to treat early-stage, HER2-positive breast cancer and in Phase I/II testing to treat HER2-positive prostate cancer. Treiger said the company is preparing to launch a Phase III trial of NeuVax to treat breast cancer but declined to disclose a start date for that trial.

Treiger wanted to see more safety data in mice, especially prior to considering the vaccine as a prophylaxis. "Safety is of paramount concern for prophylactic vaccination of healthy women who do not have cancer," he said. Specifically, he wanted to know "what happens in the breast of inoculated, pregnant mice before they begin to lactate and in postpartum mice if they aren't allowed to breast-feed their pups." Such studies would help assess whether pregnancy and/or lactation would be a contraindication for the vaccine in humans, he said.

Tuohy acknowledged that the immunized, lactating mice experienced breast inflammation and nipple irritation compared with nonimmunized, lactating mice. However, he noted that the mice went through four or five cycles of mating, giving birth and lactating without experiencing any breast tissue damage or other serious effects from are needed to determine LALBA's expression in human breast tumors and the vaccine's potential effects during and after pregnancy.

The team's first step was finding a protein that is overexpressed in a given tumor type but is rarely expressed or is absent from corresponding normal tissue. LALBA emerged as a potential breast cancer vaccine antigen because several studies in the 1980s showed that the protein was highly expressed in many breast tumor types but only expressed in healthy breast tissue during lactation, when it catalyzes the production of lactose.

Compared with vehicle, prophylactic use of a Lalba-based vaccine prevented tumorigenesis in mouse models of spontaneous breast cancer. In mouse models of induced breast cancer, prophylactic inoculation with the Lalba-based vaccine delayed disease compared with using vehicle. Therapeutic inoculation also reduced tumor growth in two mouse models of established breast cancer compared with controls.

The team found that immunized, nonlactating mice experienced no inflammation, tissue damage or other signs of an autoimmune response to the vaccine. However, immunized mice that were lactating developed inflammatory immune responses in their breasts that compromised the lactose content of their milk, which affected the weight gain of their pups compared with pups born to nonimmunized mice.

Lastly, treatment of $\mathrm{T}$ cells from healthy women with recombinant human LALBA led to the formation of LALBA-specific T cells, thereby demonstrating that the human protein is immunogenic.

Vincent Tuohy, professor of pathology and molecular medicine at the Cleveland Clinic Lerner College of Medicine of Case Western Reserve the vaccine. Thus, he thinks women receiving the vaccine would have to avoid lactation and breast-feeding but not necessarily pregnancy.

If an immunized woman were to become pregnant, "the effects of the immune response to lactation would be a nuisance and discomfort," Tuohy said. "But a woman who goes through this could ask her obstetrician or endocrinologist to terminate or prevent lactation" with hormonal treatments after she gives birth.

Nevertheless, he envisioned giving the vaccine primarily to women over the age of 40 because they are less likely to become pregnant-and thus lactate-and also have a higher risk of developing breast cancer than younger women.

He added that "the vaccine is really designed for a woman of any age who thinks she should have it, but the trade-off will always be an inability to breast-feed successfully." Thus, each woman would have to decide whether to be immunized after consulting with her physician and weighing that trade-off against her individual risk of breast cancer, he said.

"I have spoken to a lot of women about" having to trade breast-feeding for protection from potentially fatal breast cancers, Tuohy said. "Most women over 40 don't plan to have more children and said the trade-off was not an issue at all."

\section{Ounces of prevention}

In addition to the vaccine's safety profile, Treiger also wanted to know more about $L A L B A$ expression in breast tumors. He noted that of the four studies from the 1980s cited in the Tuohy team's paper, only three found 


\section{ANALYSIS}

that LALBA was highly expressed on breast tumors.

"Given the disagreement between those previous studies, a more comprehensive study is needed to get a better sense of what percentage of breast tumors express LALBA," Treiger said. That information would help assess the degree to which a LALBA-based vaccine could be used to treat or prevent breast cancer, he told SciBX.

Tuohy said a significant amount of his team's data on the LALBAbased vaccine, including complete results of safety studies in mice, were not included in the Nature Medicine paper and will be reported in a forthcoming publication.

The team's ongoing work includes comprehensive studies of LALBA expression in human breast cancer cell lines and primary breast tumors, more extensive in vitro studies of human T cell responses to LALBA and optimization of undisclosed properties of the vaccine.

The group also is testing potential antigens for a prophylactic ovarian cancer vaccine, but Tuohy declined to disclose which proteins the team is investigating.
Tuohy also declined to disclose the patent status of the findings reported in Nature Medicine. But he added, "We are looking for partners in the not-for-profit sector to get the vaccine through Phase I testing," after which the team hopes to find commercial partners.

Haas, M.J. SciBX 3(24); doi:10.1038/scibx.2010.721

Published online June 17, 2010

\section{REFERENCES}

1. Jaini, R. et al. Nat. Med.; published online May 30, 2010; doi:10.1038/nm.2161

Contact: Vincent Tuohy, Cleveland Clinic, Cleveland, Ohio e-mail: tuohyv@ccf.org

COMPANIES AND INSTITUTIONS MENTIONED

Apthera Inc., Scottsdale, Ariz.

Dendreon Corp. (NASDAQ:DNDN), Seattle, Wash.

Cleveland Clinic, Cleveland, Ohio

Cleveland Clinic Lerner College of Medicine of Case Western

Reserve University, Cleveland, Ohio

Cleveland State University, Cleveland, Ohio 